\title{
Towards molecular photochemionics
}

\author{
Margherita Venturi, ${ }^{1, \dagger}$ Vincenzo Balzani, ${ }^{1}$ Roberto Ballardini, ${ }^{2}$ \\ Alberto Credi, ${ }^{1}$ and M. Teresa Gandolfi ${ }^{1}$ \\ ${ }^{1}$ Dipartimento di Chimica “G. Ciamician”, Università di Bologna, 40126 Bologna, Italy \\ ${ }^{2}$ ISOF-CNR Institute, 40129 Bologna, Italy
}

\begin{abstract}
In the last few years there has been a great interest in developing electronics at a molecular level (molecular electronics), e.g. to construct miniaturized electric circuits that would be much smaller than the corresponding micron-scale digital logic circuits fabricated on conventional solid-state semiconductor chips. An alternative possibility to the use of electron fluxes as a means for information processing (electronics) is that of using optical beams (photonics), but up until now scarce attention has been devoted to the possibility of developing photonics at the molecular level. In this paper we review some recent achievements in the design and construction of molecular-level systems that are capable of transferring, switching, collecting, storing, and elaborating light signals. The combination of molecular photonics with chemionics can lead to a wealth of molecular-level devices capable of information processing.
\end{abstract}

\section{INTRODUCTION}

A device is something invented and constructed for a special purpose. Depending on its use, a device can be very big or very small. The progress of mankind civilization has always been related to the construction of novel devices. In the last fifty years, progressive miniaturization of the components employed for the construction of devices has resulted in outstanding achievements, particularly in the field of information technology.

The miniaturization of components for the construction of useful devices is currently pursued by the top-down approach. This approach, which is in the hands of physicists and engineers, consists in manipulating progressively smaller pieces of matter by photolithography and related techniques. It is becoming clear, however, that the top-down approach is subjected to drastic limitations for dimensions smaller than 100 nanometers [1].

An alternative strategy towards technology at the nanometer scale is offered by the bottom-up approach, which starts from molecules and builds up to nanostructures. Chemists are in an ideal position to develop bottom-up strategies for the construction of nanoscale devices since they are able to manipulate molecules, the smallest entities of matter with distinct shapes and properties. In fact, the moleculeby-molecule bottom-up strategy is nothing else than supramolecular chemistry, a discipline extensively developed in recent years [2].

\section{MOLECULAR ELECTRONICS}

In macroscopic electronic devices information processing takes place by making use of electrical signals, i.e.,

\footnotetext{
† E-mail: mventuri@ciam.unibo.it
}

by fluxes of electrons. In the last few years there has been a great interest in developing electronics at a molecular level (molecular electronics), e.g. to construct miniaturized electric circuits that would be much smaller than the corresponding micron-scale digital logic circuits fabricated on conventional solidstate semiconductor chips [3]. With this aim, a great number of investigations on electrical conductivity and electrical switching properties of molecules and supramolecular systems have been performed [4]. A schematic diagram for an AND logic gate based upon diodes and its proposed molecular implementation is shown in Figure 1 [5]. Such a gate would measure only about $3 \mathrm{~nm} \times 4 \mathrm{~nm}$, which means one million times smaller in area than the corresponding logic element fabricated on a semiconductor chip using transistor-based circuits. OR and XOR gates and a half adder have also been designed. However, much remains to be learned about the mechanisms of conductance of molecular wires and switches, as well as about the means of manipulating, bonding, and ordering them in extended circuit-like structures. If this approach is successful, it will be possible to construct ultrahigh-density molecular circuitry that would have great impact in computer science (a Pentium on a pinhead) [5].

\section{MOLECULAR PHOTONICS}

An alternative possibility to the use of electron fluxes as a means for information processing (electronics) is that of using optical beams (photonics). In several macroscopic devices electrical cables have already been replaced by optical fibers. The advantage offered by optical signals with respect to electric signals for transmission of information at the macroscopic level relies on the fact that propagating light beams of different 
(a)

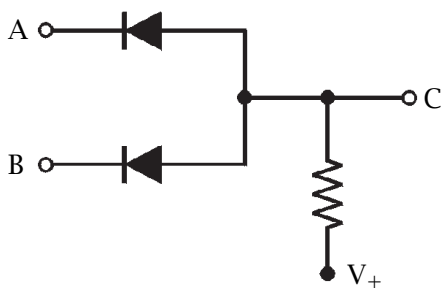

(b)

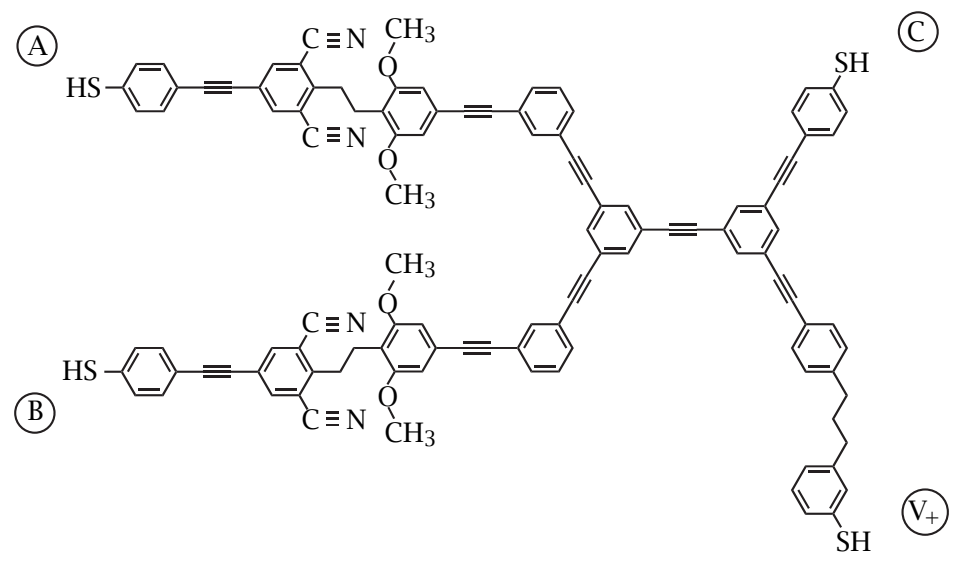

Figure 1. (a) Electronic circuit scheme, and (b) proposed molecular implementation of a diode-diode AND logic gate [5].

wavelengths in an optical fiber do not interfere, thereby allowing transportation of a large number of signals along a single fiber.

The top-down approach has allowed the construction of a variety of solid state microelectronic devices (MED) and microelectromechanical systems (MEMS) [6]. It can now be expected that the bottom-up approach will move science and technology not only from microto nanoscale, but also from electronics to photonics and chemionics since light and chemical inputs are convenient ways to power molecular-level devices and to exchange information at the molecular level. Furthermore, the bottom-up approach, taking inspiration from natural nanoscale devices, could displace the interest of scientists from solid state to solution and soft-matter. In the next few years, the bottom-up approach is likely to lead to a wealth of nanodevices in which photonics, chemionics, electronics, and mechanics will be integrated to a different extent depending on the function that the device has to perform. We shall then become accustomed with new acronyms like NED (nanoelectronic devices), NPD (nanophotonic devices), NCD (nanochemical devices), NPCD (nanophotochemical devices), NPED (nanophotoelectronic devices), NEMS (nanoelectromechanical systems), NPEMS (nanophotoelectromechanical systems), etc.

Until now scarce attention has been devoted to the possibility of developing photonics at the molecular level. One reason is that the wavelength of light in the infrared, visible and even ultraviolet light is by far greater (> $200 \mathrm{~nm}$ ) than the molecular dimensions, so that selective excitation of a specific molecule in a supramolecular array is prevented. Furthermore, devices for guiding electromagnetic radiations on a scale below the diffraction limit should be available for designing molecular-level optical circuits. Very recently, however, progress in near-field optical techniques [7] and electromagnetic energy transport in metal nanoparticle plasmon waveguides [8] seem to enable vastly increased resolution.

Regardless of the possibility of short term applications, the development of a set of components for information processing by molecular photonics seems a worthwhile investment [9]. In this paper we will review some recent achievements in the design and construction of molecular-level systems that are capable of transferring, switching, collecting, storing, and elaborating light signals, and we will show that molecular photonics and chemionics can be profitably combined to obtain systems capable of information processing in solution.

\section{DEVICES FOR TRANSFERRING LIGHT SIGNALS}

Photoinduced energy transfer is a basic process for connecting light energy inputs with a variety of optical, electrical, and mechanical functions. Several devices can be designed for delivering electronic energy, in a controlled way, to predetermined components of 
(a)
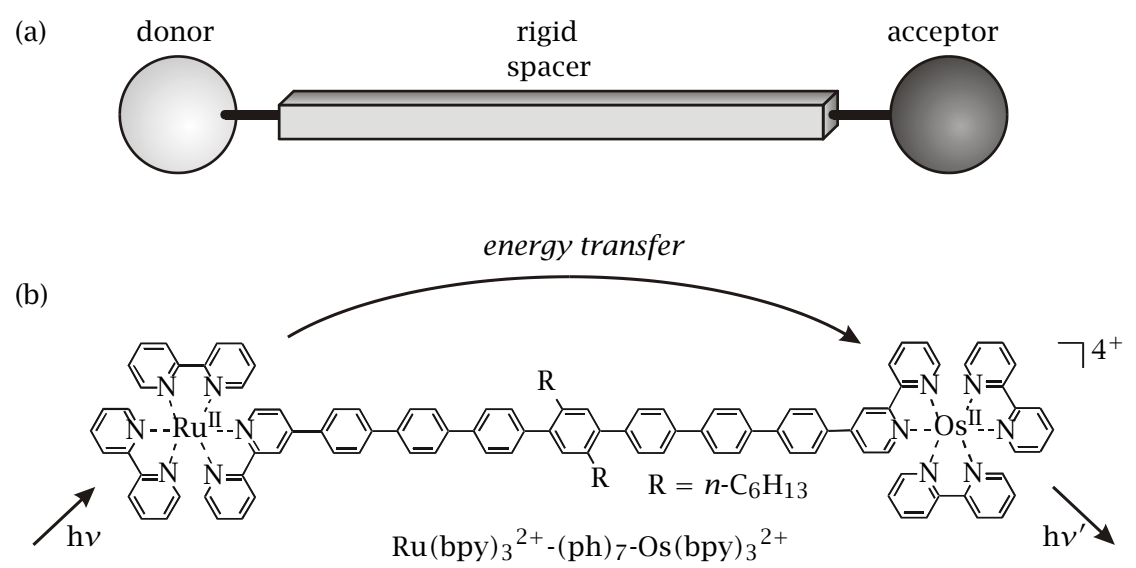

Figure 2. (a) Schematic representation of a molecular-level wire, and (b) an example of photoinduced energy-transfer process over long distances [10].

a supramolecular system. It should be pointed out that the outcoming light signal is always more or less red shifted compared with the incoming signal.

4.1. Wires. An important function at the molecular level is photoinduced energy transfer over long distances and/or along predetermined directions. This function can be obtained by linking donor and acceptor components with a rigid spacer, as illustrated in Figure 2(a). An example [10] is given by the $\left[\mathrm{Ru}(\mathrm{bpy})_{3}\right]^{2+}$. $(\mathrm{ph})_{n}$-[Os $\left.(\text { bpy })_{3}\right]^{2+}$ compounds (bpy $=2,2$ '-bipyridine; ph $=1,4$-phenylene; $n=3,5,7)$ in which excitation of the $\left[\mathrm{Ru}(\mathrm{bpy})_{3}\right]^{2+}$ unit is followed by electronic energy transfer to the ground state of the $\left[\mathrm{Os}(\mathrm{bpy})_{3}\right]^{2+}$ unit, as shown by the sensitized emission of the latter. For the compound with $n=7$ (Figure 2(b)), the rate constant for energy transfer over the $4.2 \mathrm{~nm}$ metal-to-metal distance is $1.3 \times 10^{6} \mathrm{~s}^{-1}$.

Spacers with energy levels in between those of the donor and acceptor may help energy or electron transfer (hopping mechanism). Spacers whose energy levels can be manipulated by an external stimulus can play the role of switches (vide infra).

4.2. Light harvesting antennae. In suitably designed dendrimers, electronic energy transfer can be channeled towards a specific position of the array. Compounds of this kind play the role of antennae for light harvesting. Several light harvesting dendrimers have been recently investigated, and some examples are illustrated below.

\subsubsection{A dendrimer with a metal complex as a core}

In the dendritic complex shown in Figure 3, the 2,2 '-bipyridine ligands of the $\left[\mathrm{Ru}(\mathrm{bpy})_{3}\right]^{2+}$-type core carry branches containing 1,2-dimethoxybenzene- and 2-naphthyl-type chromophoric units [11]. Since such units (as well as the core) are separated by aliphatic connections, the interchromophoric interactions are weak and the absorption spectrum of the dendrimer is substantially equal to the sum of the spectra of the chromophoric groups that are present in its structure. The three types of chromophoric groups, namely, $\left[\mathrm{Ru}(\mathrm{bpy})_{3}\right]^{2+}$, dimethoxybenzene, and naphthalene, are potentially luminescent species. In the dendrimer, however, the fluorescence of the dimethoxybenzene- and naphthyl-type units is almost completely quenched in acetonitrile solution, with concomitant sensitization of the luminescence of the $\left[\mathrm{Ru}(\mathrm{bpy})_{3}\right]^{2+}$ core $\left(\lambda_{\max }=\right.$ $610 \mathrm{~nm})$. These results show that a very efficient energytransfer process takes place converting the very short lived UV fluorescence of the aromatic units of the wedges to the long lived orange emission of the metalbased dendritic core. It should also be noted that in aerated solution the luminescence intensity of the dendrimer core is more than twice intense as that of the $\left[\mathrm{Ru}(\mathrm{bpy})_{3}\right]^{2+}$ parent compound, because the dendrimer branches protect the $\mathrm{Ru}$ - bpy based core from dioxygen quenching [12]. In conclusion, because of the very high absorbance of the naphthyl-type groups in the UV spectral region, the high energy-transfer efficiency, and the strong emission of the $\left[\mathrm{Ru}(\mathrm{bpy})_{3}\right]^{2+}$-type core, the dendrimer exhibits a strong visible emission upon UV excitation even in very dilute $\left(10^{-7} \mathrm{~mol} \mathrm{~L}^{-1}\right)$ solutions.

\subsubsection{Dendrimers with metal complexes in each branching centre}

In the past ten years a great number of dendrimers containing $\mathrm{Ru}(\mathrm{II})$ and Os(II) as metals, 2,3- and 2,5-bis(2pyridyl)pyrazine (2,3- and 2,5-dpp) as bridging ligands and 2,2'-bipyridine (bpy) and 2,2'-biquinoline (biq) as terminal ligands (Figure 4) have been carefully investigated from the photophysical viewpoint [13].

The typical strategy used to prepare dendrimers of this type is the so-called "complexes as metals and complexes as ligands" approach [14] which has allowed the 


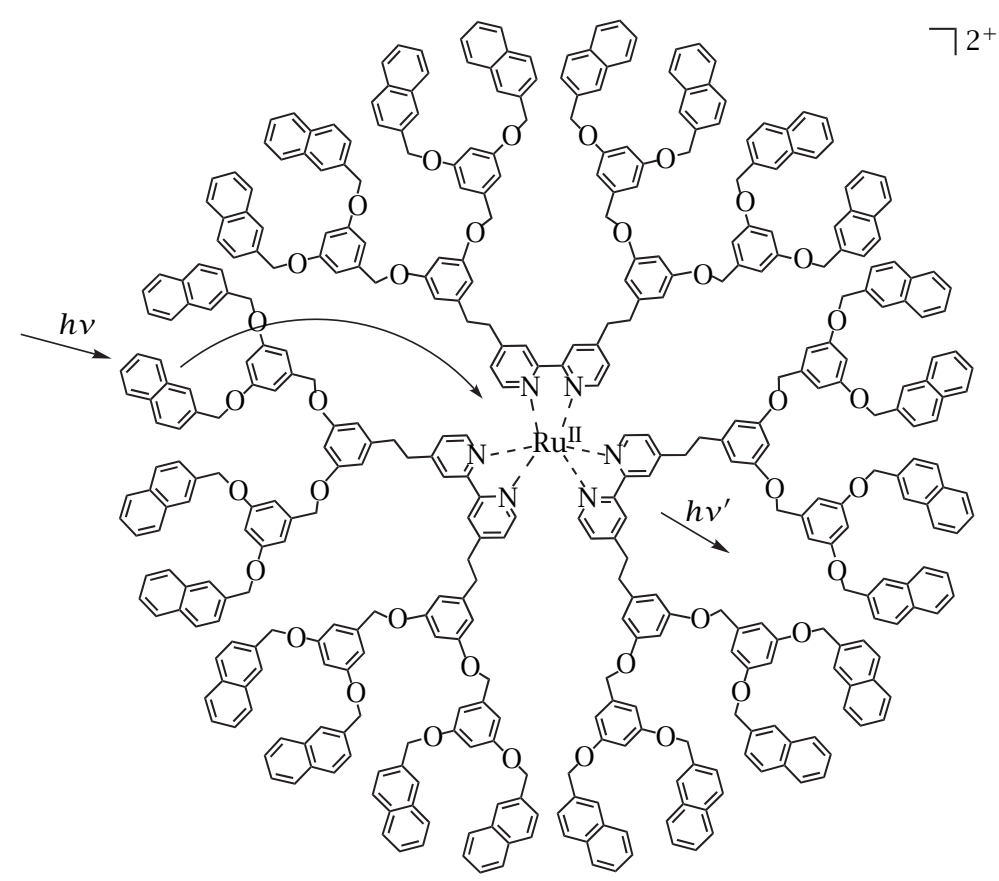

Figure 3. Antenna effect in a dendrimer [11].

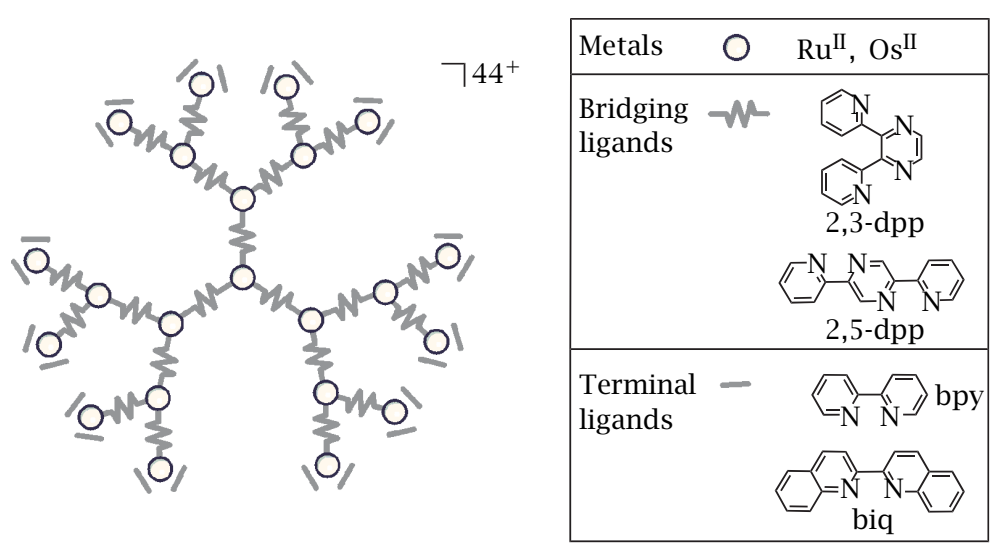

Figure 4. Schematic representation of a dendrimer containing $\mathrm{Ru}$ and/or Os complexes in each branching site [13, 14]. The formulae of the 2,3-and 2,5-bis(2-pyridyl)pyrazine (2, 3- and 2,5-dpp) bridging ligands and of the 2, 2'-bipyridine and 2, 2 '-biquinoline (bpy and biq) terminal ligands are also shown.

construction of species containing 4, 6, 10, 13 and 22 metal-based units. Such dendrimers can be viewed as ordered ensembles of $\left[\mathrm{M}(\mathrm{L})_{n}(\mathrm{BL})_{3-n}\right]^{2+}$ complexes $(\mathrm{M}=$ $\mathrm{Ru}(\mathrm{II})$ or Os(II); L = bpy or biq; BL = 2,3- or 2,5-dpp) which are known to exhibit (i) intense ligand-centered (LC) absorption bands in the UV region and moderately intense metal-to-ligand charge-transfer (MLCT) bands in the visible region, and (ii) a relatively long-lived luminescence in the red spectral region, originating from the lowest ${ }^{3}$ MLCT level.

In the dendrimers, there is only a small electronic interaction between nearby mononuclear units and therefore the absorption spectrum is practically the sum of the spectra of the constituent units. In the dendrimers of higher nuclearity, the molar absorption coefficient is huge throughout the entire UV-visible spectral region $\left(\varepsilon=202000 \mathrm{~L} \mathrm{~mol}^{-1} \mathrm{~cm}^{-1}\right.$ at $542 \mathrm{~nm}$ for the $\mathrm{Ru}(\mathrm{II})$ docosanuclear dendrimer schematically shown in Figure 4), so that most of the photochemically active part of sunlight can be absorbed.

In these dendrimers, the small but not negligible electronic interaction between nearby units is sufficient to cause a very fast energy transfer that leads to the quenching of the potentially luminescent units having higher energy ${ }^{3}$ MLCT levels and the sensitization of the luminescence of the units having lower energy ${ }^{3}$ MLCT 

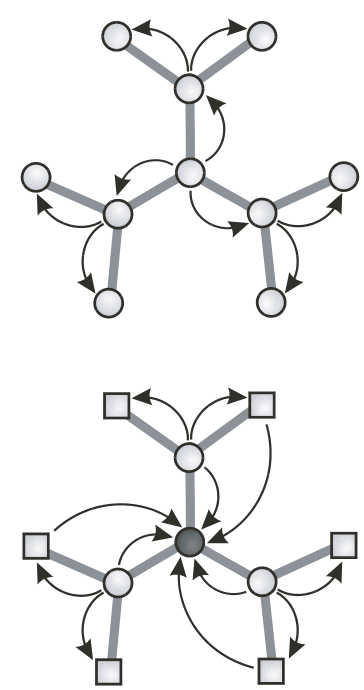
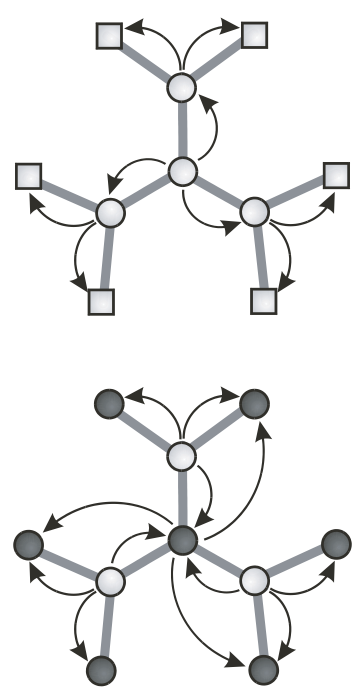
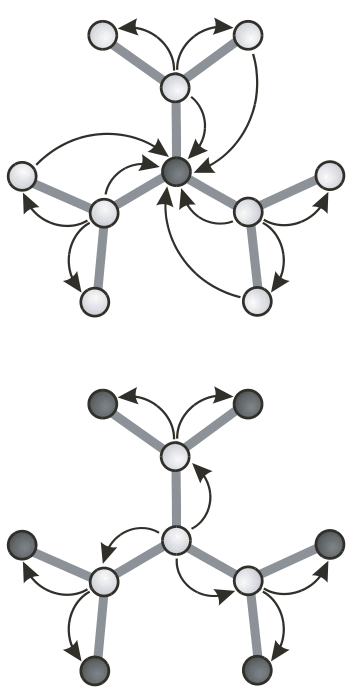

Figure 5. Schematic representation of the different energy-transfer patterns that can be obtained in decanuclear dendrimertype compounds on choosing different metals and ligands [13, 14]. The arrows indicate the energy-transfer steps; grey and black circles indicate $\mathrm{Ru}(\mathrm{II})$ and $\mathrm{Os}(\mathrm{II})$, respectively; in the peripheral positions, circles and squares indicate $\mathrm{M}(\mathrm{bpy})_{2}$ and $\mathrm{M}$ (biq) 2 components, respectively. The compounds have $20+$ electric charge.

levels. Recent studies have suggested that energy transfer between nearby units occurs within $200 \mathrm{fs}$, probably from non thermalized excited states [15].

The energy of the ${ }^{3}$ MLCT excited state of each unit depends on metal and ligands in a predictable way and the modular synthetic strategy used enables a high degree of synthetic control in terms of the nature and position of metal centers, bridging ligands, terminal ligands. Such a synthetic control translates into a high degree of control on the direction of energy flow within the dendritic array, as shown by the decanuclear compounds represented in Figure 5. On increasing nuclearity, however, a unidirectional gradient (centre-toperiphery or vice versa) for energy transfer cannot be obtained with only two types of metals [Ru(II) and Os(II)] and ligands (bpy and 2,3-dpp) [13].

\section{SWITCHING DEVICES}

5.1. An on/off function along a wire. The movement of electrons or electronic energy in a molecularlevel wire can be switched on/off by an external stimulus applied to a suitably designed unit incorporated in the wire.

In an appropriately designed compound, the external stimulus can be light. Since, by definition, switching has to be reversible, reversible photochemical reactions have to be used. Photochromic molecules are particularly useful in this regard. An example is given by the D-P-A supramolecular species investigated by Waltz et al. [16] (Figure 6), in which photoinduced energy transfer from $\mathrm{D}$ to A can be switched by photoexcitation of component $\mathrm{P}$. In such a system, the spacer $\mathrm{P}$ is a photochromic fulgide molecule which can be transformed by light in a reversible way between a closed $\mathrm{P}_{\mathrm{a}}$ and an open $\mathrm{P}_{\mathrm{b}}$ configuration. The donor $\mathrm{D}$ is an anthracene unit which can be excited at $258 \mathrm{~nm}$, and the acceptor $A$ is a coumarin molecule. When P is in its closed form $\mathrm{P}_{\mathrm{a}}$, its lowest energy level is lower than the energy level of A, so that energy transfer from D to A cannot occur (Figure 6) and the sensitized luminescence of the coumarin cannot be observed upon excitation of the anthracene moiety. However, when the P species is isomerized with $520 \mathrm{~nm}$ light to yield the $\mathrm{P}_{\mathrm{b}}$ isomer, the energy levels are in scale and the sensitized luminescence of the coumarin unit at $500 \mathrm{~nm}$ can be observed upon excitation of the anthracene unit at $258 \mathrm{~nm}$. Since light of three different wavelengths is needed and four different chromophoric units are involved, such a system is not so easy to handle and its behavior is not really on/off [16].

5.2. Plug/socket systems. Supramolecular species whose components are connected by means of non-covalent forces can be disassembled and reassembled [17] by modulating the interactions that keep the components together, thereby allowing switching of energy- or electron-transfer processes. Two-component systems of this type are reminiscent of plug/socket electrical devices and, like their macroscopic counterparts, must be characterized by (i) the possibility of connecting/disconnecting the two components in a reversible way, and (ii) the occurrence of an electronic energy or electron flow from the socket to the plug when the two components are connected (Figure 7(a)). Hydrogen-bonding interactions between ammonium ions and crown ethers are particularly convenient for constructing molecular-level 


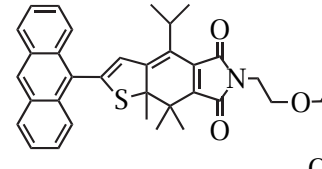

$\mathrm{D}$

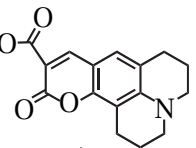

A

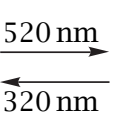

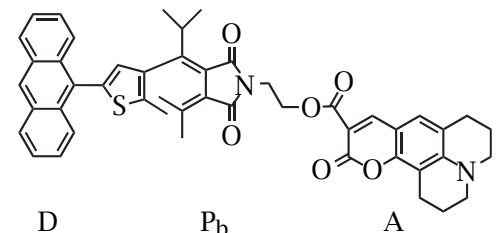

A
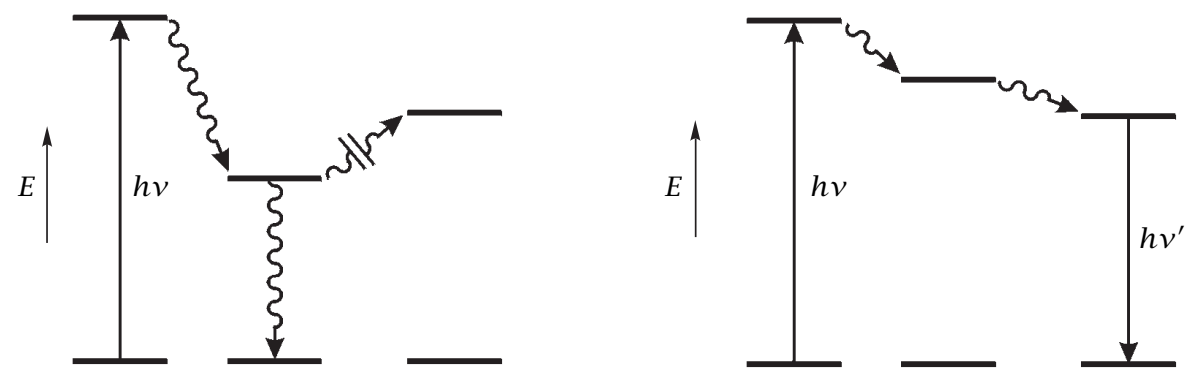

Figure 6. Switching of energy transfer from an anthracene moiety to a coumarin moiety by photoisomerization of a fulgide bridge [16].

(a)
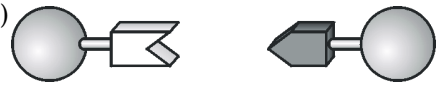

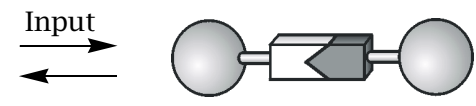

(b) $\nmid h v$

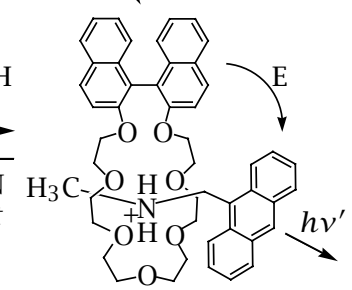

$[1 \cdot 2 \mathrm{H}]^{+}$<smiles></smiles>

2

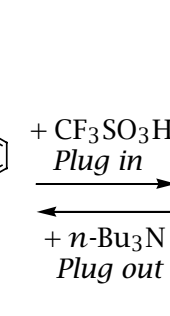

1

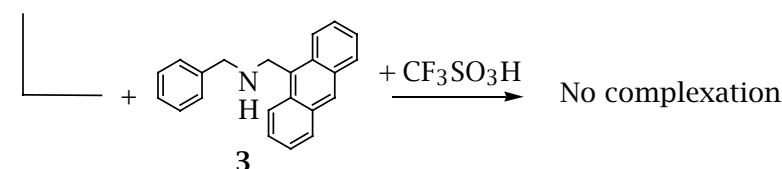

Figure 7. (a) Schematic representation of a plug-socket system, and (b) switching of energy transfer related to the acid/based-controlled plug in/plug out of the cation $[2 \mathrm{H}]^{+}$, obtained by protonation of the corresponding amine 2 , with ( \pm )-binaphthocrown ether 1 . The acid-driven threading of compound 3 does not occur [18].

plug/socket devices since they can be switched on and off quickly and reversibly by means of acid-base inputs. A plug/socket system which deals with the transfer of electronic energy is illustrated in Figure 7(b) [18]. The absorption and fluorescence spectra of a $\mathrm{CH}_{2} \mathrm{Cl}_{2}$ solution containing equal amounts of $( \pm$ )-binaphthocrown ether $\mathbf{1}$ and amine $\mathbf{2}$ indicate the absence of any interaction between the two compounds. However, addition of a stoichiometric amount of acid causes profound changes in the fluorescence behavior of the solution, namely (i) the fluorescence of $\mathbf{1}$ is quenched, and (ii) the fluorescence of $[2 \mathrm{H}]^{+}$is sensitized upon excitation with light absorbed exclusively by the crown ether.
These observations are consistent with the formation of a pseudorotaxane-type adduct wherein very efficient energy transfer takes place from the binaphthyl unit of the crown ether to the anthracene group incorporated within the dialkylammonium ion. Such a pseudorotaxane can be disassembled by the subsequent addition of a stoichiometric amount of base, thereby interrupting the photoinduced energy flow, as indicated by the fact that the initial absorption and fluorescence spectra are restored. Interestingly, the plug-in process does not take place when a plug component incompatible with the size of the socket, such as the benzyl-substituted amine 3, is employed (Figure 7(b)) [18]. 


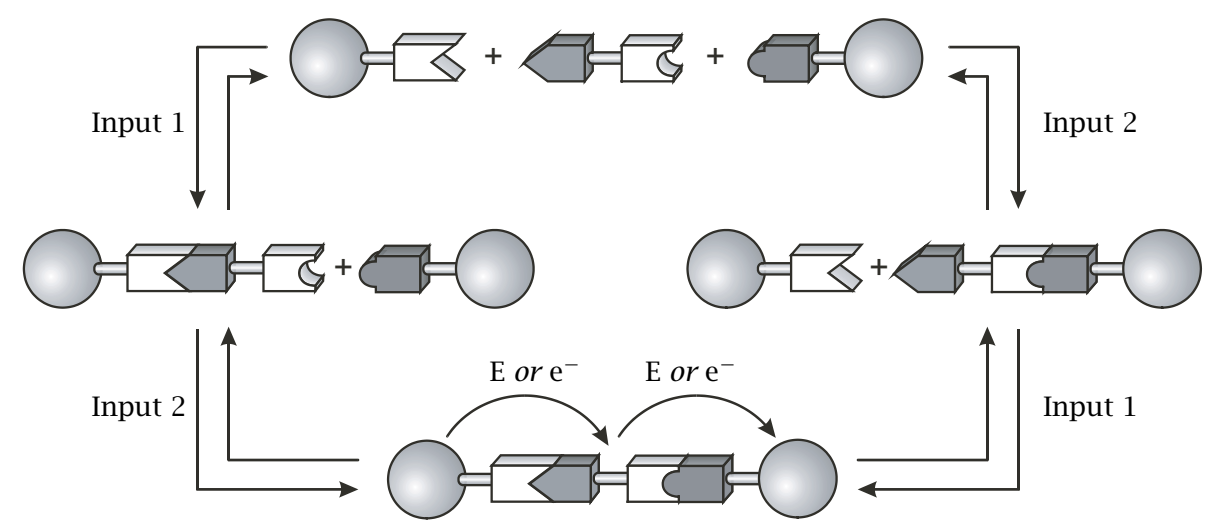

Figure 8. Schematic representation of an extension cable.

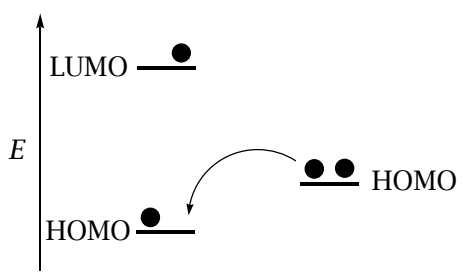

Excited fluorophore
Free receptor

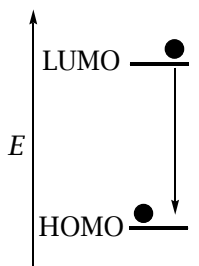

Excited fluorophore
으으 $\mathrm{HOMO}$

Bound

receptor
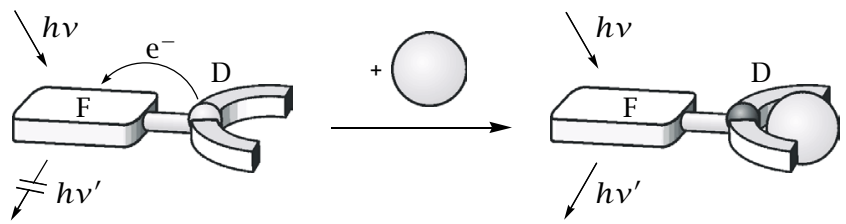

Figure 9. Schematic illustration of fluorescence switching by photoinduced electron transfer (PET) [26].

The plug/socket concept can be used to design molecular systems that mimic the function played by a macroscopic electrical extension cable. An extension cable is more complex than a plug/socket device since there are three components held together by two connections that have to be controllable reversibly and independently; in the fully connected system, an electron or energy flow must take place between the remote donor and acceptor units (Figure 8). Molecular extension cables for electron transfer have already been investigated [19]. It should not be difficult to construct molecular extension cables for energy transfer by choosing suitable components.

\section{LOGIC GATES}

Although it is not necessarily the case that the components of a molecular computer [20] will have to operate in ways analogous to those of conventional silicon-based computers, a great deal of effort has been devoted to the design, synthesis and characterization of molecular systems in which silicon-based logic can, in principle, be mimicked. Indeed, molecular-level logic functions of several types as well as very interesting molecular-based combinational logic circuits have been reported and discussed in the last few years [21-24].

Molecular switches convert input stimulation into output signals. Therefore, the principles of binary logic can be applied to the signal transduction operated by these systems. In the molecular-level switches, stimulation is usually performed by optical, electrical, or chemical inputs and the produced outputs are again optical, electrical, or chemical. It should be pointed out that input/output molecular-level processes are very common, but their logic aspects have been recognized only recently [25]. Any kind of input/output signals can be used, but most of the described examples are based on fluorescence switching caused by photoinduced electron-transfer (PET) processes, as schematically illustrated in Figure 9 [26]. In this figure, $\mathrm{F}$ is a potentially fluorescent unit (e.g., an anthracene molecule), whose fluorescent excited state is quenched by PET from the HOMO orbital of an appended electrondonor receptor, D (e.g., an amine unit). When the HOMO orbital of the electron donor is engaged by a suitable added molecule or ion (e.g., by protonation in the case 
(a)

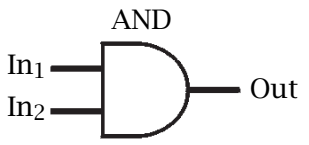

(b)

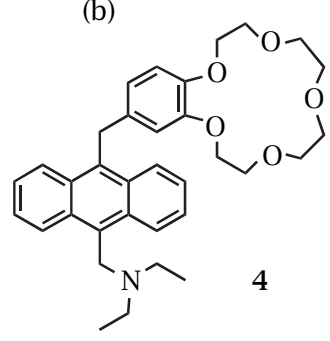

(c)

\begin{tabular}{ccc}
$\begin{array}{c}\mathrm{In}_{1} \\
\left(\mathrm{H}^{+}\right)\end{array}$ & $\begin{array}{c}\mathrm{In}_{2} \\
\left(\mathrm{Na}^{+}\right)\end{array}$ & $\begin{array}{c}\text { Out } \\
\text { (Fluo) }\end{array}$ \\
\hline 0 & 0 & 0 \\
0 & 1 & 0 \\
1 & 0 & 0 \\
1 & 1 & 1
\end{tabular}

Figure 10. (a) Symbolic representation, (b) molecular implementation, and (c) truth table of an AND logic gate based on compound 4 [28].

of an amine), fluorescence can be observed because the HOMO of the donor is lowered in energy and electron transfer can no longer occur.

These systems combine photonics with chemionics and therefore their working principles are closer to those that govern the processes of information transfer in living organisms rather than to those exploited in artificial solid state devices.

It is also important to note that fluorescence is an ideal output because of its ease of detection even at the single-molecule limit [27]. Another remarkable feature of fluorescent signals is that they do not need to be wired to operate. Light can indeed bridge the gap between the world of molecules and our macroscopic world.

For the sake of space, we will only illustrate two examples of molecular-level logic gates based on luminescence signals.

6.1. AND logic gate. The AND operator has two inputs and one output (Figure 10(a)) and in a simple electrical scheme it can be represented by two switches in series. The best examples of molecular level AND gates are those based on two chemical inputs and an optical (fluorescence) output, but examples of molecular systems able to process chemical and optical inputs or two optical inputs with AND functions are also known [22]. Figure 10(b) illustrates the case of the anthracene derivative 4 that produces a virtually perfect truth table [28]. For a $1 \times 10^{-6} \mathrm{~mol} \mathrm{~L}^{-1}$ methanol solution of 4 , the fluorescence quantum yield in the presence of $10^{-3} \mathrm{~mol} \mathrm{~L}^{-1} \mathrm{H}^{+}$and $10^{-2} \mathrm{~mol} \mathrm{~L}^{-1} \mathrm{Na}^{+}$is 0.22 (output state 1 , fourth line of the truth table, Figure $10(\mathrm{c})$ ), whereas none of the three output states 0 has quantum yield higher than 0.009. The PET quenching process involves the amine moiety in the first two states of the truth table and, of course, the crown ether in the third one.

It can be noted that the AND molecular-level logic gates shown in Figures 1 and 10(b) are completely different, not only for the chemical structure of the molecules on which they are based, but also for the chemical environment (solid state and solution, respectively) and the input and output signals. They are, in fact, different from a philosophical viewpoint: whereas the AND gate of Figure 1 is a device for solid-state electronics, that of Figure 10(b), as well as related systems like the below described XOR gate, work on chemical principles that are more similar to those ruling information transfer in living organisms.

6.2. XOR Logic Gate. The eXclusive OR (XOR) logic gate (Figure 11(a)) is particularly important because it can compare the digital state of two signals. If they are different an output 1 is given, whereas if they are the same the output is 0 . This logic operation has proven to be difficult to emulate at the molecular scale, but some examples are now available [29-31].

The first reported molecular-level XOR gate is illustrated in Figure 11 [29]. The electron-rich macrocycle 5 can be threaded by an electron-deficient wiretype molecule like $6^{2+}$. The resulting pseudorotaxane $[5 \bullet 6]^{2+}$ is held together by charge-transfer (CT) interactions. Assembling is signalled by as many as three different optical channels: (i) appearance of a red colour because of the presence of a CT absorption band in the visible region; (ii) disappearance of the blue-green fluorescence of $6^{2+}$, and (iii) disappearance of the UV fluorescence of 5 with $\lambda_{\max }=343 \mathrm{~nm}$ (the disappearance of the two fluorescent signals is due to the presence of the lower lying CT state in the pseudorotaxane). For the XOR function, protons and $n-\mathrm{Bu}_{3} \mathrm{~N}$ are the inputs and the fluorescence of $\mathbf{5}$ at $343 \mathrm{~nm}$ is the output. The working mechanism and truth table of this system are illustrated schematically in Figures 11(b) and 11(c), respectively. As mentioned above, in the absence of the two inputs the fluorescence of $\mathbf{5}$ is quenched in the pseudorotaxane (output 0). When the $\mathrm{Bu}_{3} \mathrm{~N}$ input alone is applied, the pseudorotaxane dethreads because of the formation of a stronger CT interaction between 
(a)

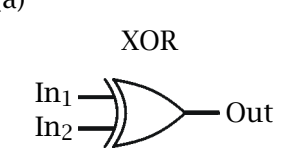

(c)

\begin{tabular}{ccc}
$\begin{array}{c}\mathrm{In}_{1} \\
\left(\mathrm{H}^{+}\right)\end{array}$ & $\begin{array}{c}\mathrm{In}_{2} \\
\left(n-\mathrm{Bu}_{3} \mathrm{~N}\right)\end{array}$ & $\begin{array}{c}\text { Out } \\
(\text { Fluo })\end{array}$ \\
\hline 0 & 0 & 0 \\
0 & 1 & 1 \\
1 & 0 & 1 \\
1 & 1 & 0
\end{tabular}

(b)
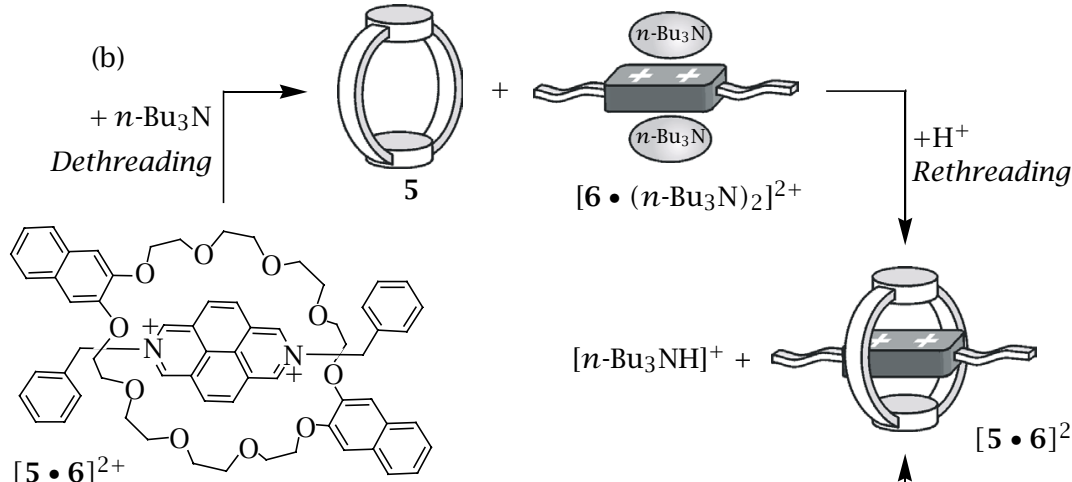

$[5 \cdot 6]^{2+}$
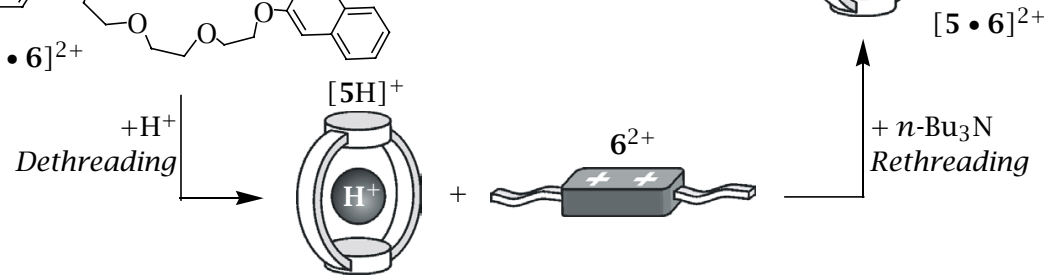

Figure 11. (a) Symbolic representation, (b) molecular implementation and working mechanism, and (c) truth table of an XOR logic gate based on compounds 5 and $6^{2+}$ [29].

the amine and $6^{2+}$. Under such conditions, 5 is free and its fluorescence is not quenched (output 1). Application of the $\mathrm{H}^{+}$input causes protonation of $\mathbf{5}$ and, again, dethreading of the pseudorotaxane. Since protonation of $\mathbf{5}$ (presumably at the aliphatic ether oxygens) does not perturb its emission compared to the neutral form, the $\mathrm{H}^{+}$input 1 causes an output 1 . Therefore the output achieves logic state 1 in the two situations in which exclusively one of the two inputs is present. However, when both inputs are applied in stoichiometric amounts, acid-base neutralisation results, the pseudorotaxane remains intact and the $343 \mathrm{~nm}$ emission is quenched (output 0).

\section{CONCLUSION}

In this perspective article, we have shown that molecular-level systems capable of transferring, switching, collecting, storing, and elaborating light signals have recently been investigated.

We have also pointed out that the bottom-up approach and the combination of photonics and chemionics displace the interest of scientists from solid state to solution and soft-matter.

The molecular electronic approach in which molecules are used as simple circuit components, exemplified by the AND gate shown in Figure 1, has the potential advantage of being strictly related to the paradigms of current microelectronics technology [32]. On the other hand, the "photochemionic" approach illustrated by the systems described above, can lead to a better understanding of natural information processing systems and can perhaps lead to a new "soft" technology. It is difficult, at the present stage, to predict which one of these two strategies will have the greater technological impact. These and other questions regarding the advent of molecular computers (e.g., serial or parallel architectures, solid-state or "wet") represent one of the big challenges of nanotechnology.

\section{ACKNOWLEDGEMENTS}

Financial support from EU (project HPRN-CT-200000029), the University of Bologna (Funds for Selected Research Topics), and MIUR (Supramolecular Devices Project) is gratefully acknowledged.

\section{REFERENCES}

[1] R. W. Keyes, Proc. IEEE 89 (2001), 227.

[2] (a) F. Vögtle, Supramolecular Chemistry, Wiley, Chichester, 1991; (b) Frontiers in Supramolecular Organic Chemistry and Photochemistry (H.J. Schneider and H. Dürr, eds.), VCH, Weinheim, 1991; (c) Supramolecular Chemistry (V. Balzani and L. De Cola, eds.), Kluwer, Dordrecht, 1992; (d) Transition Metals in Supramolecular Chemistry (L. Fabbrizzi and A. Poggi, eds.), Kluwer, Dordrecht, 1994; (e) Comprehensive Supramolecular Chemistry (J. L. Atwood, J. E. D. Davies, D. D. Macnicol, and F. Vögtle, eds.), Pergamon Press, Oxford, 1996, Vol. 1-10; (f) Physical Supramolecular Chemistry (L. Echegoyen and A. E. Kaifer, eds.), Kluwer, Dordrecht, 1996; (g) Modular Chemistry (J. Michl, ed.), Kluwer, Dordrecht, 1997; (h) Transition Metals in Supramolecular Chemistry (J.-P. Sauvage, ed.), Wiley, New York, 1999; (i) Supramolecular Science: Where it is and Where it is Going (R. Ungaro and E. Dalcanale, eds.), Kluwer, Dordrecht, 1999; (j) H.-J. Schneider and A. Yatsimirsky, Principles and Methods in Supramolecular Chemistry, Wiley, Chichester, 2000; (k) J. W. Steed and J. L. Atwood, Supramolecular Chemistry, Wiley, Chichester, 2000; (l) G. R. Newkome, C. N. Moorefield, 
and F. Vögtle, Dendrimers and Dendrons, WileyVCH, Weinheim, 2001; (m) S. J. Rowan, S. J. Cantrill, G. R. L. Cousins, J. K. M. Sanders, and J. F. Stoddart, Angew. Chem. Int. Ed. 41 (2002), 898; (n) J.-M. Lehn, Proc. Natl. Acad. Sci. USA 99 (2002), 4763.

[3] M. A. Reed, Proc. IEEE 87 (1999), 652.

[4] For a few representative papers, see: (a) R. M. Metzger, Acc. Chem. Res. 32 (1999), 950; (b) C. Joachim, J. K. Gimzewski, and A. Aviram, Nature 408 (2000), 541; (c) J. M. Tour, Acc. Chem. Res. 33 (2000), 791; (d) R. E. Holmlin, R. F. Ismagilov, R. Haag, V. Mujica, M. A. Ratner, M. A. Rampi, and G. M. Whitesides, Angew. Chem. Int. Ed. 40 (2001), 2316; (e) X. D. Cui, A. Primak, X. Zarate, J. Tomfohr, O. F. Sankey, A. L. Moore, T. A. Moore, D. Gust, G. Harris, and S. M. Lindsay, Science 294 (2001), 571; (f) F.-R. F. Fan, J. Yang, L. Cai, D. W. Price Jr., S. M. Dirk, D. V. Kosynkin, Y. Yao, A. M. Rawlett, J. M. Tour, and A. J. Bard, J. Am. Chem. Soc. 124 (2002), 5550; (g) Y. Luo, C. P. Collier, J. O. Jeppesen, K. A. Nielsen, E. DeIonno, G. Ho, J. Perkins, H.-R. Tseng, T. Yamamoto, J. F. Stoddart, and J. R. Heath, ChemPhysChem 3 (2002), 519.

[5] J. C. Ellenbogen and J. C. Love, Proc. IEEE 88 (2000), 386.

[6] (a) I. Amato, Science 282 (1998), 402; (b) D. Barrow, J. Cefai, and S. Taylor, Chem. Ind. 2 (1999), 591; (c) J. W. Judy, Smart Mater. Struct. 10 (2001), 1115.

[7] (a) K. Lieberman, S. Harush, A. Lewis, and R. Kopelman, Science 247 (1990), 59; (b) D. A. Higgins, D. A. Vanden Bout, J. Kerimo, and P. F. Barbara, J. Phys. Chem. 100 (1996), 13794; (c) M. Irie and K. Matsuda, Electron Transfer in Chemistry, Vol. 5 (V. Balzani, ed.), Wiley-VCH, Weinheim, 2001, p. 215; (d) R. Hillenbrand, T. Taubner, and F. Keilmann, Nature 418 (2002), 159.

[8] J. A. Maier, M. L. Brongersma, P. G. Kik, S. Meltzer, A. A. G. Requicha, and H. A. Atwater, Adv. Mater. 13 (2001), 1501.

[9] V. Balzani, A. Credi, and M. Venturi, Molecular Devices and Machines - A Journey into the Nanoworld, Wiley-VCH, Weinheim, 2003.

[10] B. Schliche, P. Belser, L. De Cola, E. Sabbioni, and V. Balzani, J. Am. Chem. Soc. 121 (1999), 4207, and unpublished results.

[11] M. Plevoets, F. Vögtle, L. De Cola, and V. Balzani, New J. Chem. 23 (1999), 63.

[12] The dendrimer branches protect the excited state of the core also from quenching by other species: F. Vögtle, M. Plevoets, M. Nieger, G. C. Azzellini, A. Credi, L. De Cola, V. De Marchis, M. Venturi, and V. Balzani, J. Am. Chem. Soc. 121 (1999), 6290.

[13] (a) V. Balzani, S. Campagna, G. Denti, A. Juris, S. Serroni, and M. Venturi, Acc. Chem. Res. 31 (1998), 26; (b) V. Balzani, P. Ceroni, A. Juris, M. Venturi, S. Campagna, F. Puntotiero, and S. Serroni, Coord. Chem. Rev. 219 (2001), 545.
[14] G. Denti, S. Serroni, S. Campagna, A. Juris, M. Ciano, and V. Balzani, Perspectives in Coordination Chemistry (A. F. Williams, C. Floriani, and A. E. Merbach, eds.), VCH, Basel, 1992, p. 153.

[15] H. B. Baudin, J. Davidsson, S. Serroni, A. Juris, V. Balzani, S. Campagna, and L. Hammarstrom, J. Phys. Chem. A 106 (2002), 4312.

[16] J. Walz, K. Ulrich, H. Port, H. C. Wolf, J. Wonner, and F. Effenberger, Chem. Phys. Lett. 213 (1993), 321.

[17] V. Balzani, A. Credi, and M. Venturi, Proc. Natl. Acad. Sci. USA 99 (2002), 4814.

[18] E. Ishow, A. Credi, V. Balzani, F. Spadola, and L. Mandolini, Chem. Eur. J. 5 (1999), 984.

[19] R. Ballardini, V. Balzani, M. Clemente-Leon, A. Credi, M. T. Gandolfi, E. Ishow, J. Perkins, J. F. Stoddart, H. R. Tseng, and S. Wenger, J. Am. Chem. Soc. 124 (2002), 12786.

[20] (a) D. Rouvray, Chem. Brit. 36 (2000) 46; (b) P. Ball, Nature 406 (2000), 118.

[21] V. Balzani, A. Credi, and M. Venturi, Supramolecular Science: Where it is and Where it is Going ( $\mathrm{R}$. Ungaro and E. Dalcanale, eds.), Kluwer, Dordrecht, 1999, p. 1.

[22] (a) A. P. de Silva, N. D. McClenaghan, and C. P. McCoy, Electron Transfer in Chemistry, Vol. 5 (V. Balzani, ed.), Wiley-VCH, Weinheim, 2001, p. 156; (b) A. P. de Silva, N. D. McClenaghan, and C. P. McCoy, Molecular Switches (B. L. Feringa, ed.), WileyVCH, Weinheim, 2001, p. 339.

[23] A. R. Pease and J. F. Stoddart, Struct. Bond. 99 (2001), 189.

[24] F. M. Raymo, Adv. Mater. 14 (2002), 401.

[25] (a) A. P. de Silva, H. Q. N. Gunaratne, and C. P. McCoy, Nature 364 (1993), 42; (b) A. P. de Silva and N. D. McClenaghan, Chem. Eur. J. 8 (2002), 4935.

[26] A. P. de Silva, H. Q. N. Gunaratne, T. Gunnlaugsson, A. J. M. Huxley, C. P. McCoy, J. T. Rademacher, and T. E. Rice, Chem. Rev. 97 (1997), 1515.

[27] (a) S. Weiss, Science 283 (1999), 1676; (b) Single Molecule Detection in Solution (Ch. Zander, J. Enderlein, and R. A. Keller, eds.), Wiley-VCH, Berlin, 2002.

[28] A. P. de Silva, H. Q. N. Gunaratne, and C. P. McCoy, J. Am. Chem. Soc. 119 (1997), 7891.

[29] A. Credi, V. Balzani, S. J. Langford, and J. F. Stoddart, J. Am. Chem. Soc. 119 (1997), 2679.

[30] F. Pina, M. J. Melo, M. Maestri, P. Passaniti, and V. Balzani, J. Am. Chem. Soc. 122 (2000), 4496.

[31] A. P. de Silva and N. D. McClenaghan, J. Am. Chem. Soc. 122 (2000), 3965.

[32] For an assessment of the state-of-the-art and future requirements of semiconductor technology, see: The International Technology Roadmap for Semiconductors (ITRS), 2001 ed., available at http://public.itrs.net. 


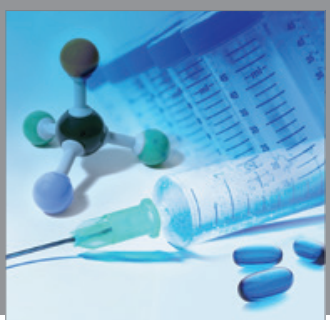

International Journal of

Medicinal Chemistry

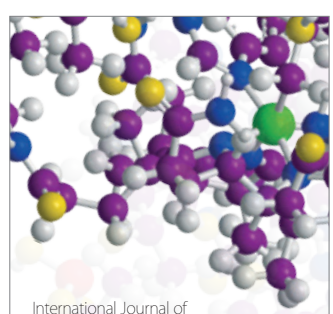

Carbohydrate Chemistry

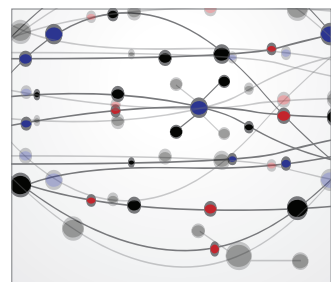

The Scientific World Journal
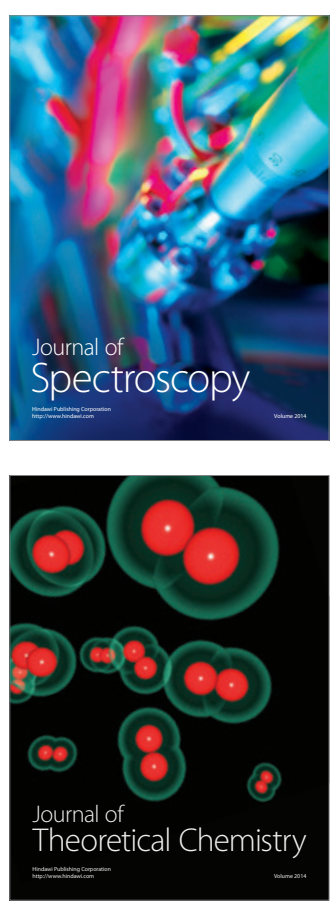
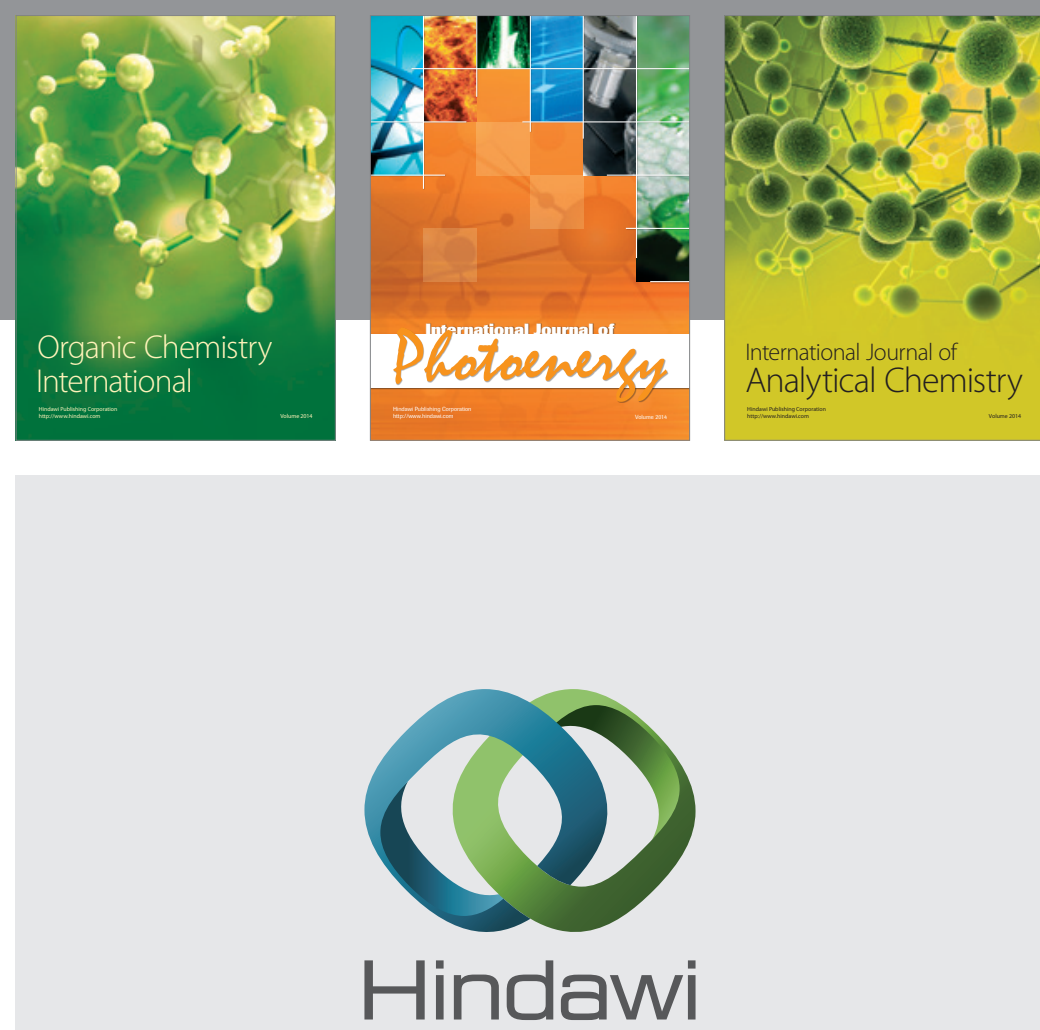

Submit your manuscripts at

http://www.hindawi.com
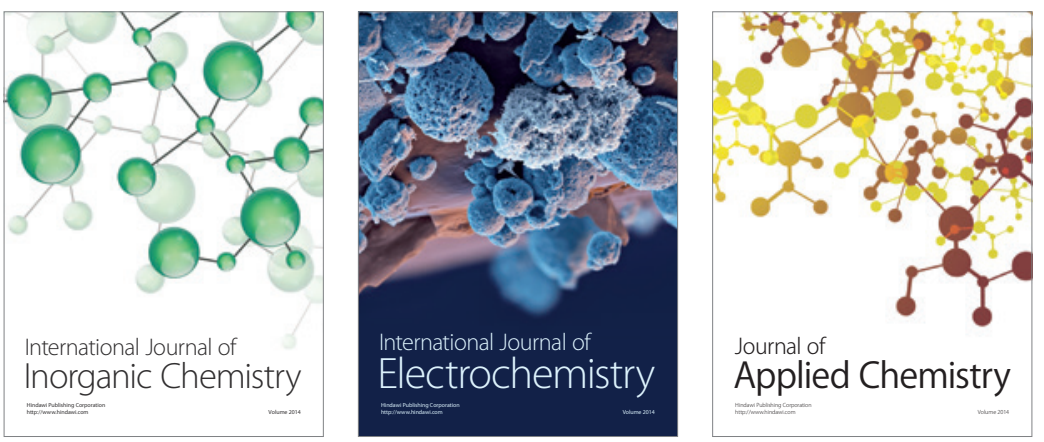

Journal of

Applied Chemistry
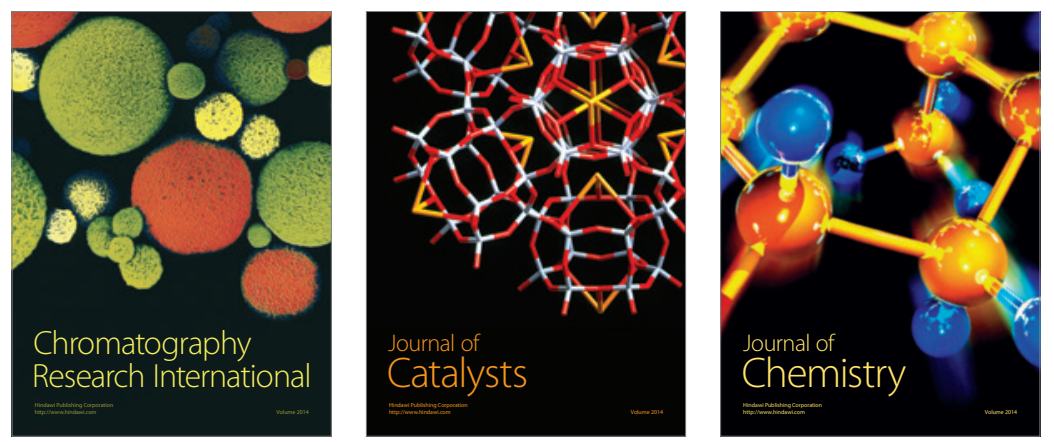
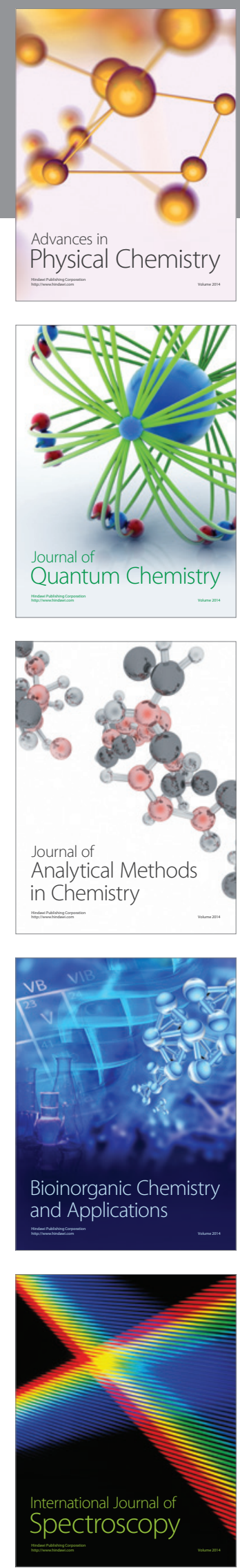